\title{
Join and Meet Operations for Type-2 Fuzzy Sets With Non-Convex Secondary Memberships
}

\author{
Gonzalo Ruiz, Hani Hagras, Fellow, IEEE, Hctor Pomares, Ignacio Rojas, Asscociate Member, IEEE, \\ and Humberto Bustince, Senior Member, IEEE
}

\begin{abstract}
In this paper we will present two theorems for the join and meet operations for general type-2 fuzzy sets with arbitrary secondary memberships, which can be non-convex and/or non-normal type-1 fuzzy sets. These results will be used to derive the join and meet operations of the more general descriptions of interval type-2 fuzzy sets presented in [1], where the secondary grades can be non-convex. Hence, this work will help to explore the potential of type-2 fuzzy logic systems which use the general forms of interval type-2 fuzzy sets which are not equivalent to interval valued fuzzy sets. Several examples for both general type- 2 and the more general forms of interval type-2 fuzzy sets are presented.
\end{abstract}

Index Terms-type-2 fuzzy logic, general type-2 fuzzy logic, non-convex fuzzy sets.

\section{INTRODUCTION}

G ENERAL Type-2 Fuzzy Sets (GT2FSs) are characterised by secondary memberships which take any value between 0 and 1 (unlike Interval Type-2 Fuzzy Sets (IT2FSs), whose secondary memberships are either 0 or 1$)$. The meet and join operations for General Type-2 Fuzzy Sets (GT2FSs), which represent the intersection and union for these sets respectively, are based on the Extension Principle by Zadeh [2] as a generalisation of the intersection and union for type-1 fuzzy sets. In 2001, the initial work by Karnik and Mendel presented in [3] a simplified procedure to compute these operations for GT2FSs, although it depended on the condition that the secondary grades of type-2 fuzzy sets were normal and convex type-1 fuzzy sets. This work was later generalised by Coupland [4] to incorporate non-normal sets by borrowing some methods (Weiler-Atherton, Modified Weiler Atherton, Bentley-Ottmann Plane Sweep Algorithm, etc.) from the field of computational geometry; yet convexity remained a necessary condition. More recent works [5], [6], [7] studied the geometrical properties of some GT2FSs to find closed formulas or approximations for the join and meet operations in some specific cases. However, to the best of the authors knowledge, considering arbitrary secondary grades, which can be non-convex, has not been addressed to date.

Recent developments in type-2 fuzzy logic have changed the perception researchers have of Interval-Type 2 Fuzzy Sets (IT2FSs). IT2FSs are type-2 fuzzy sets which uncertainty is equally distributed in the third dimension (also called secondary membership), and thus, these secondary membership are either 0 or 1, unlike GT2FSs, which uncertainty in the third dimension is not equally weighted and the distribution can be an arbitrary type-1 fuzzy set. When IT2FSs were initially defined in [8], all the theory and operations were based on the specific case where IT2FSs are equivalent to Interval Valued Fuzzy Sets (IVFSs). However, it has been recently shown that IT2FSs are more general than IVFSs [1]. Hence, in order to derive the theory of these general forms of Interval Type-2 Fuzzy Logic Systems (IT2FLS) (which employ IT2FSs which are not equivalent to IVFSs) it is necessary to develop the meet and join operations of GT2FSs with non-convex secondary memberships, and then, particularise it to the case of IT2FSs, which have secondary grades equal to either 0 or 1 .

Hence, in this paper, we will be finding the join and meet operations for GT2FSs where secondary memberships are arbitrary type-1 sets, and hence can be non-convex and/or non-normal. This will be used to derive the join and meet operations of IT2FSs where the secondary grades are nonconvex sets.

The structure of this paper is as follows: Section II will present preliminaries in order to provide some basic background. Section III will present two theorems for the join and meet operations for GT2FSs making no assumptions about their normality or convexity. In Section IV we will apply our results to the general forms of IT2FSs presented in [1]. Section $\mathrm{V}$ will present examples of the two theorems applied to GT2FSs with normal and convex secondary memberships, GT2FSs with non-convex and non-normal secondary memberships, and all general forms of IT2FSs (including type-1 sets and interval-valued fuzzy sets) as presented in [1]. Conclusions and future work are presented in Section VI.

\section{Preliminaries}

Type-2 fuzzy sets are an extension of type-1 fuzzy sets. While a type-1 fuzzy set $F$ is characterized by a type1 membership function (MF) $\mu_{F}(x)$ (where $x \in X$ and $\left.\mu_{F}(x) \in[0,1]\right)$, a type- 2 set $\tilde{F}$ is characterized by a type$2 \operatorname{MF} \mu_{\tilde{F}}(x, u)$, where $x \in X$ and $u \in J_{x} \subseteq[0,1]$, i.e. [8], [9]:

$$
\tilde{F}=\left\{\left((x, u), \mu_{\tilde{F}}(x, u)\right) \mid \forall x \in X, \forall u \in J_{x} \subseteq[0,1]\right\}
$$

$\tilde{F}$ can also be expressed as follows [8]:

$$
\tilde{F}=\int_{x \in X} \int_{u \in J_{x}} \mu_{\tilde{F}}(x, u) /(x, u) \quad J_{x} \subseteq[0,1]
$$

Where $\iint$ denotes aggregation over all admissible $x$ and $u . J_{x}$ is called the primary membership of $x$ in $\tilde{F}$. At each value of $x$, say $x=x^{\prime}$, the 2-D plane whose axes are $u$ and $\mu_{\tilde{F}}\left(x^{\prime}, u\right)$ is called a vertical slice of $\tilde{F}$ [8]. A secondary membership 
function is a vertical slice of $\tilde{F}$. It is, $\mu_{\tilde{F}}\left(x=x^{\prime}, u\right)$, for $x^{\prime} \in X$ and $\forall u \in J_{x^{\prime}} \subseteq[0,1]$, [2], i.e.

$$
\mu_{\tilde{F}}\left(x=x^{\prime}, u\right) \equiv \mu_{\tilde{F}}\left(x^{\prime}\right)=\int_{u \in J_{x^{\prime}}} f_{x^{\prime}}(u) / u \quad J_{x^{\prime}} \subseteq[0,1]
$$

Because $\forall x^{\prime} \in X$, the prime notation on $\mu_{\tilde{F}}\left(x^{\prime}\right)$ is dropped and $\mu_{\tilde{F}}(x)$ is referred to as a secondary membership function [8], [9]; it is a type-1 fuzzy set which is also referred to as a secondary set [8]. If $\forall x \in X$ the secondary membership function is an interval type-1 set where $f_{x}(u)=1 \forall u \in J_{x}$, (i.e. $\mu_{\tilde{F}}(x, u)=1$ ), the type- 2 set $\tilde{F}$ is referred to as an interval type- 2 fuzzy set. It should be noted that the notation we use here does not imply that $J_{x}$ should only consider the values where $f_{x}(u)$ is greater than zero. In this work we consider $J_{x}=[0,1]$ to simplify the representation.

\section{JOIN AND MEET OPERATIONS FOR GT2FSS With NON-CONVEX SECONDARY MEMBERSHIPS}

In this section we will present two theorems for the join and meet operations for GT2FSs with non-convex secondary memberships.

\section{A. The Join Operation}

Definition 1: Let $\tilde{F}_{1}$ and $\tilde{F}_{2}$ be two type-2 fuzzy sets in a universe of discourse $X$. Let $\mu_{\tilde{F}_{1}}(x)$ and $\mu_{\tilde{F}_{2}}(x)$ denote the membership grades of $\tilde{F}_{1}$ and $F_{2}$, respectively, at $x \in X$. Then, for each $x \in X$, using minimum t-norm and maximum t-conorm, the union set $\tilde{F}_{1} \cup \tilde{F}_{2}$, which is characterised by its membership grade $\mu_{\tilde{F}_{1} \cup \tilde{F}_{2}}(x, \theta)$ is given by the join operation on $\mu_{\tilde{F}_{1}}(x)$ and $\mu_{\tilde{F}_{2}}(x)$, and is as follows ${ }^{1}$ :

$$
\begin{aligned}
& \mu_{\tilde{F}_{1} \cup \tilde{F}_{2}}(x, \theta)=\left(\mu_{\tilde{F}_{1}}(x) \sqcup \mu_{\tilde{F}_{2}}(x)\right)(\theta)= \\
& =\sup _{v \in[0, \theta]}\left\{f_{1}(v)\right\} \wedge\left(f_{1}(\theta) \vee f_{2}(\theta)\right) \wedge \sup _{w \in[0, \theta]}\left\{f_{2}(w)\right\} \\
& x \in X
\end{aligned}
$$

Such that $v \vee w=\theta$.

Theorem 1: The union operation on two type-2 fuzzy sets defined in [3] using minimum t-norm is equivalent to the union defined in Equation (4).

Proof of Theorem 1: Let the join operation be performed on two type-2 fuzzy sets, denoted $\tilde{F}_{1}$ and $\tilde{F}_{2}$, in a universe of discourse $X$. The membership grades at $x \in X$ of $\tilde{F}_{1}$ and $\tilde{F}_{2}$ are denoted as $\mu_{\tilde{F}_{1}}(x)$ and $\mu_{\tilde{F}_{2}}(x)$, respectively, which are fuzzy sets defined in $V, W \subseteq[0,1]$, and are as in Equation (3). According to [3], the union of two type-2 fuzzy sets, denoted as $\tilde{F}_{1} \cup \tilde{F}_{2}$, is given by the join operation between $\tilde{F}_{1}$ and $\tilde{F}_{2}$ as follows:

$$
\begin{aligned}
& \tilde{F}_{1} \cup \tilde{F}_{2} \leftrightarrow \mu_{\tilde{F}_{1} \sqcup \tilde{F}_{2}}(x)= \\
& =\int_{v \in V} \int_{w \in W}\left(f_{1}(v) * f_{2}(w) /(v \vee w) \quad x \in X\right.
\end{aligned}
$$

\footnotetext{
${ }^{1}$ It should be noted that Equation (4) has some similarity to Equation (10) in [10] (see also [11]) as both equations refer to the join operation of general type-2 fuzzy sets. However, the representation of Equation (4) is quite different to simplify the computations and analysis.
}

Where $*$ indicates the minimum t-norm (hence $*$ will be replaced by $\wedge$ in the rest of the paper) and $\vee$ indicates the maximum t-conorm.

Thus, any element $\theta=(v \vee w)$ in the primary membership of $\tilde{F}_{1} \cup \tilde{F}_{2}$ can be obtained by any of the following cases:

1) Case 1: if $v$ is any value between 0 and $\theta$, and $w=\theta$; i.e, $\{(v, w) \mid v \leq \theta$ and $w=\theta\} \rightarrow(v \vee w)=(v \vee \theta)=\theta$. This condition is equivalent to state that $v \in[0, \theta]$ and $w=\theta$.

2) Case 2: if $w$ is any value between 0 and $\theta$, and $v=\theta$; i.e, $\{(v, w) \mid w \leq \theta$ and $v=\theta\} \rightarrow(v \vee w)=(\theta \vee w=\theta)$. This condition is equivalent to state that $w \in[0, \theta]$ and $v=\theta$.

The membership value associated with $\theta$ can be obtained by applying the minimum t-norm on the secondary grades $f_{1}(v)$ and $f_{2}(w)$ where $v$ and $w$ are as described in Cases 1 and 2; hence, $\mu_{\tilde{F}_{1} \cup \tilde{F}_{2}}(x, \theta)=f_{1}(v) \wedge f_{2}(w)$.

It is important to note that if more than one pair $\{v, w\}$ result in the same $\theta=(v \vee w)$ but with different membership grade $\mu_{\tilde{F}_{1} \cup \tilde{F}_{2}}(x, \theta)=f_{1}(v) \wedge f_{2}(w)$, then we keep the maximum membership grade obtained from all $\{v \vee w\}$ pairs. Hence, $\mu_{\tilde{F}_{1} \cup \tilde{F}_{2}}(x, \theta)=f_{1}(v) \wedge f_{2}(w)$ is obtained by the following steps:

- Step 1: calculate $\phi_{1}(\theta)$, where:

$$
\phi_{1}(\theta)=\sup _{v \in[0, \theta]}\left\{f_{1}(v) \wedge f_{2}(\theta)\right\}
$$

According to the notation used in [10], Equation (6) would be $f_{1}^{L}(\theta) \wedge f_{2}(\theta)$. See [10] for this notation.

- Step 2: calculate $\phi_{2}(\theta)$, where:

$$
\phi_{2}(\theta)=\sup _{w \in[0, \theta]}\left\{f_{1}(\theta) \wedge f_{2}(w)\right\}
$$

According to the notation used in [10], Equation (7) would be $f_{1}(\theta) \wedge f_{2}^{L}(\theta)$.

- Step 3: calculate $\mu_{\tilde{F}_{1} \cup \tilde{F}_{2}}(x, \theta)$ where:

$$
\mu_{\tilde{F}_{1} \cup \tilde{F}_{2}}(x, \theta)=\phi_{1}(\theta) \vee \phi_{2}(\theta)
$$

$f_{1}(\theta)$ and $f_{2}(\theta)$ are fixed as $\theta$ is fixed. Hence, $f_{1}(\theta)$ and $f_{2}(\theta)$ will not be considered for the suprema calculation. Consequently, we can rewrite Equation (6) and Equation (7) as Equation (9) and Equation (10), respectively, and combine them in Equation (11).

$$
\begin{gathered}
\phi_{1}(\theta)=\sup _{v \in[0, \theta]}\left\{f_{1}(v)\right\} \wedge f_{2}(\theta) \\
\phi_{2}(\theta)=f_{1}(\theta) \wedge \sup _{w \in[0, \theta]}\left\{f_{2}(w)\right\} \\
\mu_{\tilde{F}_{1} \cup \tilde{F}_{2}}(x, \theta)=\left(\sup _{v \in[0, \theta]}\left\{f_{1}(v)\right\} \wedge f_{2}(\theta)\right) \\
\vee\left(f_{1}(\theta) \wedge \sup _{w \in[0, \theta]}\left\{f_{2}(w)\right\}\right)
\end{gathered}
$$

Using four labels denoted as $A_{1}, B_{1}, C_{1}$ and $D_{1}$ as illustrated in Equation (12), Equation (11) can be rewritten as in Equation (13).

$$
\begin{aligned}
& \mu_{\tilde{F}_{1} \cup \tilde{F}_{2}}(x, \theta)=(\underbrace{\sup _{v \in[0, \theta]}\left\{f_{1}(v)\right\}}_{A_{1}} \wedge \underbrace{f_{2}(\theta)}_{B_{1}}) \vee \\
& (\underbrace{f_{1}(\theta)}_{C_{1}} \wedge \underbrace{\sup _{w \in[0, \theta]}\left\{f_{2}(w)\right\}}_{D_{1}})
\end{aligned}
$$




$$
\mu_{\tilde{F}_{1} \cup \tilde{F}_{2}}(x, \theta)=\left(A_{1} \wedge B_{1}\right) \vee\left(C_{1} \wedge D_{1}\right)
$$

The distributive property of minimum and maximum operations allows us to re-write the right hand side of Equation (13) as follows:

$$
\begin{aligned}
& \left(A_{1} \wedge B_{1}\right) \vee\left(C_{1} \wedge D_{1}\right)= \\
& \left(A_{1} \vee C_{1}\right) \wedge\left(A_{1} \vee D_{1}\right) \wedge\left(B_{1} \vee C_{1}\right) \wedge\left(B_{1} \vee D_{1}\right)
\end{aligned}
$$

By substituting Equation (14) in Equation (13) and replacing the labels denoted as $A_{1}, B_{1}, C_{1}$ and $D_{1}$, Equation (14) can be written as below:

$$
\begin{aligned}
\mu_{\tilde{F}_{1} \cup \tilde{F}_{2}}(x, \theta) & =\left(\sup _{v \in[0, \theta]}\left\{f_{1}(v)\right\} \vee f_{1}(\theta)\right) \wedge \\
& \left(\sup _{v \in[0, \theta]}\left\{f_{1}(v)\right\} \vee \sup _{w \in[0, \theta]}\left\{f_{2}(w)\right\}\right) \wedge \\
& \left(f_{2}(\theta) \vee f_{1}(\theta)\right) \wedge\left(f_{2}(\theta) \vee \sup _{w \in[0, \theta]}\left\{f_{2}(w)\right\}\right)
\end{aligned}
$$

Using four labels denoted as $A_{2}, B_{2}, C_{2}$ and $D_{2}$ as illustrated in Equation (16), Equation (15) can be re-written as shown in Equation (17).

$$
\begin{aligned}
& \mu_{\tilde{F}_{1} \cup \tilde{F}_{2}}(x, \theta)=\underbrace{\left(\sup _{v \in[0, \theta]}\left\{f_{1}(v)\right\} \vee f_{1}(\theta)\right)}_{A_{2}} \wedge \\
&(\underbrace{\left.\sup _{v \in[0, \theta]}\left\{f_{1}(v)\right\} \vee \sup _{w \in[0, \theta]}\left\{f_{2}(w)\right\}\right)}_{C_{2}} \\
&(\underbrace{\left.f_{2}(\theta) \vee f_{1}(\theta)\right)}_{B_{2}} \wedge(\underbrace{f_{2}(\theta)}_{\left.\tilde{B}_{2}(\theta) \vee \sup _{w \in[0, \theta]}\left\{f_{2}(w)\right\}\right)} \\
& \mu_{\tilde{F}_{1} \cup \tilde{F}_{2}}(x, \theta)=A_{2} \wedge B_{2} \wedge C_{2} \wedge D_{2}
\end{aligned}
$$

It is worthwhile to analyse two of the terms in Equation (17), which are $A_{2}$ and $D_{2}$, separately:

$$
A_{2}=\sup _{v \in[0, \theta]}\left\{f_{1}(v)\right\} \vee f_{1}(\theta)
$$

In the term $A_{2}$ shown in Equation (18), it is important to note that the value $f_{1}(\theta)$ is included in the value $\sup _{v \in[0, \theta]}\left\{f_{1}(v)\right\}$, as the value $v=\theta$ belongs to the interval $v \in[0, \theta]$. Hence, the maximum $f_{1}(\theta) \vee \sup _{v \in[0, \theta]}\left\{f_{1}(v)\right\}$ will always be represented in the value $\sup _{v \in[0, \theta]}\left\{f_{1}(v)\right\}$, regardless of the value of $\theta$ and the shape of the function $f_{1}(v)$. Consequently, term $A_{2}$ in Equation (18) can be written as $A_{2}^{\prime}$ as shown in Equation (19):

$$
\begin{aligned}
& A_{2}=\sup _{v \in[0, \theta]}\left\{f_{1}(v)\right\} \vee f_{1}(\theta)=\sup _{v \in[0, \theta]}\left\{f_{1}(v)\right\}=A_{2}^{\prime} \\
& \rightarrow f_{1}(\theta) \in\left\{f_{1}(v) \mid v \in[0, \theta]\right\} \rightarrow f_{1}(\theta) \leq \sup _{v \in[0, \theta]}\left\{f_{1}(v)\right\}
\end{aligned}
$$

Similarly, we will use the abovementioned approach for the term $D_{2}$ in Equation (16):

$$
D_{2}=f_{2}(\theta) \vee \sup _{w \in[0, \theta]}\left\{f_{2}(w)\right\}
$$

Analogously, $D_{2}$ is equivalent to $D_{2}^{\prime}$ :

$D_{2}=\sup _{w \in[0, \theta]}\left\{f_{2}(w)\right\} \vee f_{2}(\theta)=\sup _{w \in[0, \theta]}\left\{f_{2}(w)\right\}=D_{2}^{\prime}$

$\rightarrow f_{2}(\theta) \in\left\{f_{2}(w) \mid w \in[0, \theta]\right\} \rightarrow f_{2}(\theta) \leq \sup _{w \in[0, \theta]}\left\{f_{2}(w)\right\}$
By using $A_{2}^{\prime}$ instead of $A_{2}$, and using $D_{2}^{\prime}$ instead of $D_{2}$ in Equation (17), we have Equation (22) as follows:

$$
\mu_{\tilde{F}_{1} \cup \tilde{F}_{2}}(x, \theta)=A_{2}^{\prime} \wedge B_{2} \wedge C_{2} \wedge D_{2}^{\prime}
$$

Substituting each label $A_{2}^{\prime}, B_{2}, C_{2}$ and $D_{2}^{\prime}$ with their corresponding contents, we obtain Equation (23):

$$
\begin{aligned}
\mu_{\tilde{F}_{1} \cup \tilde{F}_{2}}(x, \theta)= & \sup _{v \in[0, \theta]}\left\{f_{1}(v)\right\} \wedge \\
& \left(\sup _{v \in[0, \theta]}\left\{f_{1}(v)\right\} \vee \sup _{w \in[0, \theta]}\left\{f_{2}(w)\right\}\right) \wedge \\
& \left(f_{2}(\theta) \vee f_{1}(\theta)\right) \wedge \sup _{w \in[0, \theta]}\left\{f_{2}(w)\right\}
\end{aligned}
$$

In order to simplify the notations in the equation we will again label each term in Equation (23) separately as shown below:

$$
\begin{aligned}
\mu_{\tilde{F}_{1} \cup \tilde{F}_{2}}(x, \theta) & =\underbrace{\sup _{v \in[0, \theta]}\left\{f_{1}(v)\right\}}_{A_{3}} \wedge \\
& (\underbrace{\sup _{v \in[0, \theta]}\left\{f_{1}(v)\right\}}_{A_{3}} \vee \underbrace{\sup _{w \in[0, \theta]}\left\{f_{2}(w)\right\}}_{B_{3}}) \wedge \\
& \underbrace{\left(f_{2}(\theta) \vee f_{1}(\theta)\right)}_{C_{3}} \wedge \underbrace{\sup p_{w \in[0, \theta]}\left\{f_{2}(w)\right\}}_{B_{3}}
\end{aligned}
$$

Using three labels denoted as $A_{3}, B_{3}$ and $C_{3}$ as illustrated in Equation (24), Equation (23) can be expressed as shown in Equation (25).

$$
\mu_{\tilde{F_{1}} \cup \tilde{F_{2}}}(x, \theta)=A_{3} \wedge\left(A_{3} \vee B_{3}\right) \wedge C_{3} \wedge B_{3}
$$

We will focus on the partial expression $A_{3} \wedge\left(A_{3} \vee B_{3}\right)$ in Equation (25). Using the fact that $a \wedge(a \vee b)=a$ for any real numbers $a$ and $b$, then $A_{3} \wedge\left(A_{3} \vee B_{3}\right)=A_{3}$, and Equation(25) becomes Equation (26). Substituting each label by its content, we have Equation (27).

$$
\mu_{\tilde{F}_{1} \cup \tilde{F}_{2}}(x, \theta)=A_{3} \wedge\left(A_{3} \vee B_{3}\right) \wedge C_{3} \wedge B_{3}=A_{3} \wedge C_{3} \wedge B_{3}
$$

$\mu_{\tilde{F}_{1} \cup \tilde{F}_{2}}(x, \theta)=$

$\sup _{v \in[0, \theta]}\left\{f_{1}(v)\right\} \wedge\left(f_{1}(\theta) \vee f_{2}(\theta)\right) \wedge \sup _{w \in[0, \theta]}\left\{f_{2}(w)\right\}$

$x \in X$

Equation (27) is the same as Equation (4) and this concludes the proof of Theorem 1. This equation is the final result for the join operation performed on two type- 2 fuzzy sets, $\tilde{F}_{1}$ and $\tilde{F}_{2}$, for each $x \in X$. It is important to note that this result is obtained without any assumption regarding the normality or convexity of the secondary grades, denoted $f_{1}(v)$ and $f_{2}(w)$, that belong to the fuzzy sets $\tilde{F}_{1}$ and $\tilde{F}_{2}$, respectively.

\section{B. The Meet Operation}

Definition 2: Let $\tilde{F}_{1}$ and $\tilde{F}_{2}$ be two type-2 fuzzy sets in a universe of discourse $X$. Let $\mu_{\tilde{F}_{L}}(x)$ and $\mu_{\tilde{F}_{2}}(x)$ denote the membership grades of $\tilde{F}_{1}$ and $\tilde{F}_{2}$, respectively, at $x \in X$. Then, using minimum t-norm, the intersection set $\tilde{F}_{1} \cap \tilde{F}_{2}$, which is characterised by its membership function $\mu_{\tilde{F}_{1} \cap \tilde{F}_{2}}$, is 
given by the meet operation on $\mu_{\tilde{F}_{1}}(x)$ and $\mu_{\tilde{F}_{2}}(x)$, and is as follows ${ }^{2}$ :

$$
\begin{aligned}
& \mu_{\tilde{F}_{1} \cap \tilde{F}_{2}}(x, \theta)=\left(\mu_{\tilde{F}_{1}}(x) \sqcap \mu_{\tilde{F}_{2}}(x)\right)(\theta)= \\
& =\sup _{v \in[\theta, 1]}\left\{f_{1}(v)\right\} \wedge\left(f_{1}(\theta) \vee f_{2}(\theta)\right) \wedge \sup _{w \in[\theta, 1]}\left\{f_{2}(w)\right\} \\
& x \in X
\end{aligned}
$$

Theorem 2: The intersection operation on two type-2 fuzzy sets defined in [3] using minimum t-norm is equivalent to the intersection defined in Equation (28).

Proof of Theorem 2: Proof of theorem 2 is very similar to the proof of theorem 1 . In this case, any element $\theta$ in the primary membership of $\tilde{F}_{1} \cap \tilde{F}_{2}$ is of the form $\theta=(v \wedge w)$, and can be obtained by any of the following two cases:

1) Case 1: $v \in[\theta, 1]$ and $w=\theta$.

2) Case 2: $w \in[\theta, 1]$ and $v=\theta$.

The rest of the proof is exactly the same as the one for the join operation, but changing the intervals $v \in[0, \theta]$ and $w \in[0, \theta]$ by $v \in[\theta, 1]$ and $w \in[\theta, 1]$, respectively. The final result will be as in Equation (28).

\section{JOIN AND MEET OPERATIONS FOR THE GENERAL DESCRIPTIONS OF IT2FSS}

In this section, we will focus on the particular case where $f_{1}(v)$ and $f_{2}(w)$ are either 0 or 1 and their supports are nonempty closed sets. In other words, we will focus on the general descriptions of IT2FSs, as presented in [1]. We will obtain specific versions of Equation (4) and Equation (28) when sets are general forms of IT2FSs. It is important to note that all examples in [1] satisfy that the supports of $f_{1}(v)$ and $f_{2}(w)$ are non-empty closed sets.

Let $g_{1}(\theta)=\sup _{v \in[0, \theta]}\left\{f_{1}(v)\right\}$. For a given value of $\theta, g_{1}(\theta)$ is the maximum value that the function $f_{1}(v)$ has attained for all values of $v$ lower than or equal to $\theta$, i.e., $\forall v \leq \theta$. Let $v_{1}$ be the infimum of the support of $f_{1}$. Hence, for all $\theta<v_{1}$ :

$$
g_{1}(\theta)=\sup _{v \in[0, \theta]}\left\{f_{1}(v)\right\}=\sup _{v \in[0, \theta]}\{0\}=0 \quad \forall \theta<v_{1}
$$

For values $\theta \geq v_{1}$, as $f_{1}\left(v_{1}\right)=1$, the following stands:

$$
\begin{aligned}
& g_{1}(\theta)=\sup _{v \in[0, \theta]}\left\{f_{1}(v)\right\} \\
& =f_{1}\left(v_{1}\right) \vee \sup _{v \in[0, \theta]}\left\{f_{1}(v)\right\}= \\
& v \neq v_{1} \\
& 1 \vee \sup _{v \in[0, \theta]}\left\{f_{1}(v)\right\}=1 \quad \forall \theta \geq v_{1} \\
& v \neq v_{1}
\end{aligned}
$$

Hence, combining Equations (29) and (30):

$$
g_{1}(\theta)=\sup _{v \in[0, \theta]}\left\{f_{1}(v)\right\}= \begin{cases}0 & \forall \theta<v_{1} \\ 1 & \forall \theta \geq v_{1}\end{cases}
$$

Analogously, let $g_{2}(\theta)=\sup _{w \in[0, \theta]}\left\{f_{2}(w)\right\}$ and let $w_{1}$ be the infimum of the support of $f_{2}$. Hence:

$$
g_{2}(\theta)=\sup _{w \in[0, \theta]}\left\{f_{2}(w)\right\}= \begin{cases}0 & \forall \theta<w_{1} \\ 1 & \forall \theta \geq w_{1}\end{cases}
$$

\footnotetext{
${ }^{2}$ It should be noted that Equation (28) has some similarity to Equation (11) in [10] as both equations refer to the meet operation of general type-2 fuzzy sets. However, the representation of Equation (28) is different to simplify the computations and analysis.
}

Let $g(\theta)=g_{1}(\theta) \wedge g_{2}(\theta)$. Combining Equations (31) and (32):

$$
g(\theta)= \begin{cases}0 & \theta<\max \left(v_{1}, w_{1}\right) \\ 1 & \theta \geq \max \left(v_{1}, w_{1}\right)\end{cases}
$$

Considering the definition of $g(\theta)$, we can rewrite Equation (4) as:

$$
\mu_{\tilde{F}_{1} \cup \tilde{F}_{2}}(x, \theta)=g(\theta) \wedge\left(f_{1}(\theta) \vee f_{2}(\theta)\right)
$$

Combining Equations (33) and (34):

$$
\mu_{\tilde{F}_{1} \cup \tilde{F}_{2}}(x, \theta)=\left\{\begin{array}{l}
0 \quad \theta<\max \left(v_{1}, w_{1}\right) \\
f_{1}(\theta) \vee f_{2}(\theta) \quad \theta \geq \max \left(v_{1}, w_{1}\right)
\end{array}\right.
$$

Let $v_{\text {end }}$ and $w_{\text {end }}$ be the supremum of the supports of $f_{1}$ and $f_{2}$, respectively. Hence, $f_{1}(v)=0 \quad \forall v>v_{\text {end }}$ and $f_{2}(w)=0 \quad \forall w>w_{\text {end }}$. Therefore, the term $f_{1}(\theta) \vee f_{2}(\theta)$ will be $0 \quad \forall \theta>\max \left(v_{\text {end }}, w_{\text {end }}\right)$. Consequently we can rewrite Equation (35) as:

$$
\mu_{\tilde{F_{1}} \cup \tilde{F}_{2}}(x, \theta)=\left\{\begin{array}{c}
f_{1}(\theta) \vee f_{2}(\theta) \\
\quad \theta \in\left[\max \left(v_{1}, w_{1}\right), \max \left(v_{\text {end }}, w_{\text {end }}\right)\right] \\
0 \quad \text { elsewhere }
\end{array}\right.
$$

Now let's consider the case of the meet operation. In this case, let $g_{1}(\theta)=\sup _{v \in[\theta, 1]}\left\{f_{1}(v)\right\}$. Given a value of $\theta>v_{\text {end }}$ :

$$
g_{1}(\theta)=\sup _{v \in[\theta, 1]}\left\{f_{1}(\theta)\right\}=\sup _{v \in[\theta, 1]}\{0\}=0 \quad \forall \theta>v_{\text {end }}
$$

For values $\theta \leq v_{\text {end }}$, as $f_{1}\left(v_{\text {end }}\right)=1$, the following stands:

$$
\begin{aligned}
& g_{1}(\theta)=\sup _{v \in[\theta, 1]}\left\{f_{1}(v)\right\}=f_{1}\left(v_{\text {end }}\right) \vee \sup _{v} \in[\theta, 1] \\
& v \neq v_{\text {end }} \\
&=1 \vee \sup _{\substack{v \in[\theta, 1] \\
v \neq v_{\text {end }}}}\left\{f_{1}(v)\right\}=1 \quad \forall \theta \leq v_{\text {end }}
\end{aligned}
$$

Hence, combining Equations (37) and (38):

$$
g_{1}(\theta)=\sup _{v \in[\theta, 1]}\left\{f_{1}(v)\right\}= \begin{cases}1 & \forall \theta \leq v_{\text {end }} \\ 0 & \forall \theta>v_{\text {end }}\end{cases}
$$

A similar expression can be found for $g_{2}(\theta)=$ $\sup _{w \in[\theta, 1]}\left\{f_{2}(w)\right\}$.

$$
g_{2}(\theta)=\sup _{w \in[\theta, 1]}\left\{f_{2}(w)\right\}= \begin{cases}1 & \forall \theta \leq w_{\text {end }} \\ 0 & \forall \theta>w_{\text {end }}\end{cases}
$$

Let $g(\theta)=g_{1}(\theta) \wedge g_{2}(\theta)$. We can rewrite Equation (28) as:

$$
\mu_{\tilde{F}_{1} \cap \tilde{F}_{2}}(x, \theta)=g(\theta) \wedge\left(f_{1}(\theta) \vee f_{2}(\theta)\right)
$$

Considering $g(\theta)=g_{1}(\theta) \wedge g_{2}(\theta)$ and using Equations (39) and (40), we can rewrite Equation (28) as follows:

$$
\mu_{\tilde{F}_{1} \cap \tilde{F}_{2}}(x, \theta)=\left\{\begin{array}{l}
f_{1}(\theta) \vee f_{2}(\theta) \quad \theta \leq \min \left(v_{\text {end }}, w_{\text {end }}\right) \\
0 \quad \theta>\min \left(v_{\text {end }}, w_{\text {end }}\right)
\end{array}\right.
$$

By definition of $v_{1}$ and $w_{1}, f_{1}(v)=0 \quad \forall v<v_{1}$ and $f_{2}(w)=0 \quad \forall w<w_{1}$. Therefore, the term $f_{1}(\theta) \vee f_{2}(\theta)$ will be $0 \quad \forall \theta<\min \left(v_{1}, w_{1}\right)$. Consequently we can rewrite Equation (42) as:

$\mu_{\tilde{F}_{1} \cap \tilde{F}_{2}}(x, \theta)=\left\{\begin{array}{l}f_{1}(\theta) \vee f_{2}(\theta) \\ \quad \theta \in\left[\min \left(v_{1}, w_{1}\right), \min \left(v_{\text {end }}, w_{\text {end }}\right)\right] \\ 0 \quad \text { elsewhere }\end{array}\right.$ 

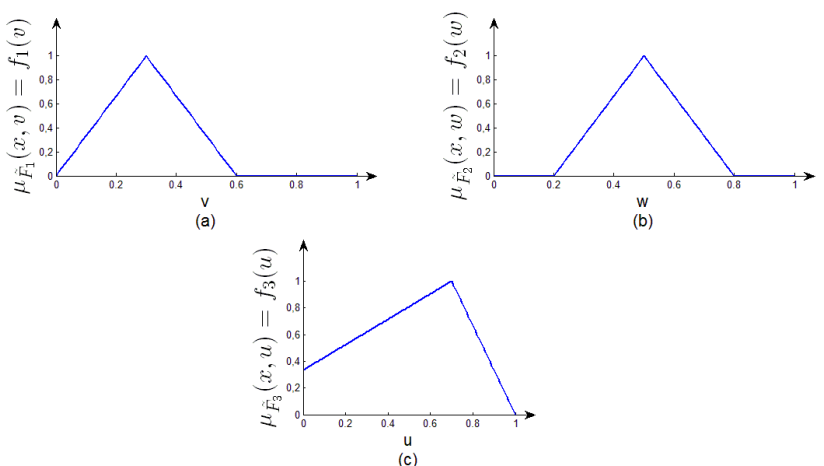

Fig. 1. Vertical slices of three GT2FSs to perform the join operation.

It is worthwhile to highlight that Equations (36) and (43) lead to the well-known results of the join and meet when the involved sets are type-1 sets or IVFSs. For the join in type-1 sets, as $v_{1}=v_{\text {end }}$ and $w_{1}=w_{\text {end }}$, then $\theta$ is non zero only when $\theta=\max \left(v_{1}, w_{1}\right)$, so $f_{1}(\theta) \vee f_{2}(\theta)$ is a singleton placed a this value $\theta=\max \left(v_{1}, w_{1}\right)$. An analogous reasoning for the meet operation, given $\theta=\min \left(v_{1}, w_{1}\right)$, leads to a singleton placed at this $\theta=\min \left(v_{1}, w_{1}\right)$.

For the case of IVFSs, as $f_{1}$ and $f_{2}$ have continuous supports, then $f_{1}(\theta) \vee f_{2}(\theta)$ will also be continuous in $\theta \in\left[\max \left(v_{1}, w_{1}\right), \max \left(v_{\text {end }}, w_{\text {end }}\right)\right]$ for the join, and $\theta \in$ $\left[\min \left(v_{1}, w_{1}\right), \min \left(v_{e n d}, w_{\text {end }}\right)\right]$ for the meet, regardless of the relative positions of $v_{1}, w_{1}, v_{\text {end }}$ and $w_{\text {end }}$, thus leading to the well-known equations for IVFSs:

$\mu_{\tilde{F}_{1} \cup \tilde{F}_{2}}(x, \theta)= \begin{cases}1 & \theta \in\left[\max \left(v_{1}, w_{1}\right), \max \left(v_{\text {end }}, w_{\text {end }}\right)\right] \\ 0 & \text { elsewhere }\end{cases}$

$\mu_{\tilde{F}_{1} \cap \tilde{F}_{2}}(x, \theta)= \begin{cases}1 & \theta \in\left[\min \left(v_{1}, w_{1}\right), \min \left(v_{\text {end }}, w_{\text {end }}\right)\right] \\ 0 & \text { elsewhere }\end{cases}$

\section{EXAMPLES OF THE JOIN AND MEET OPERATIONS}

In this section we will present several examples of the join and meet operations on different kinds of type- 2 fuzzy sets.

\section{A. Join and Meet Operations for GT2FSs with normal and convex secondary memberships}

In this section, we will show that our approach for the join and meet operations on two GT2FSs presented in Equation (4) and Equation (28) give consistent results when compared with the existing approaches where secondary grades are normal and convex type- 1 fuzzy sets. We have used as a benchmark the examples presented in [12] (page 493, fig. 5), which are shown in Figure 1, so that we can compare the results achieved by Theorem 1 and Theorem 2 to the results achieved in [12].

First of all, we will perform the join operation on the first two sets given in Figure 1(a) and Figure 1(b); and secondly, we will perform the join operation on the resulting set and the set given in Figure 1(c).

Let $g_{1}(\theta)=\sup _{v \in[0, \theta]}\left\{f_{1}(v)\right\}$. It can be proven that, for any convex and normal secondary grade $f_{1}$ having its
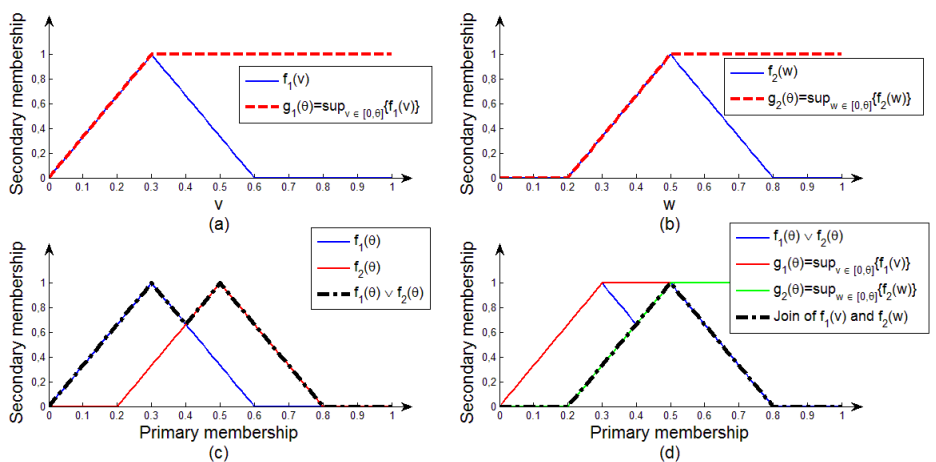

(d)

Fig. 2. (a) $f_{1}(v)$ and $g_{1}(\theta)$. (b) $f_{2}(w)$ and $g_{2}(\theta)$. (c) $f_{1}(\theta), f_{2}(\theta)$ and $f_{1}(\theta) \vee$ $f_{2}(\theta)$. (d) All terms and join result.
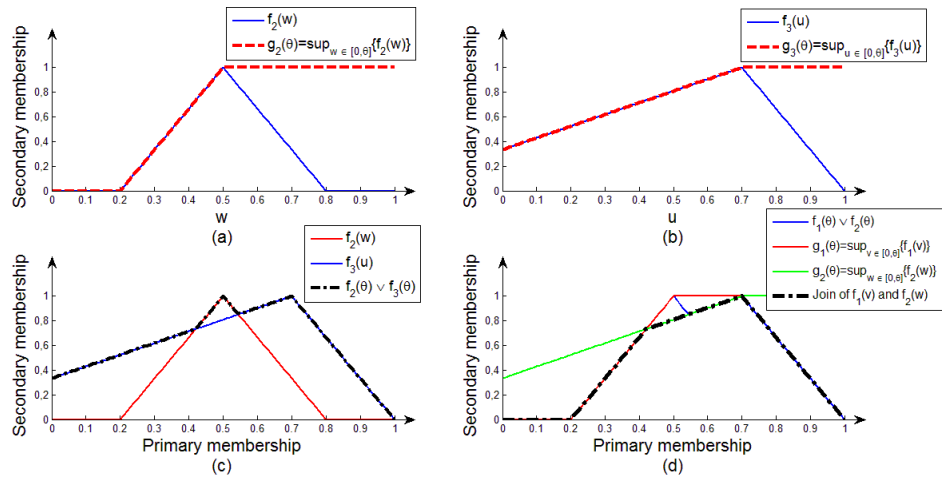

Fig. 3. (a) $f_{2}(w)$ and $g_{2}(\theta)$. (b) $f_{3}(u)$ and $g_{3}(\theta)$. (c) $f_{2}(\theta), f_{3}(\theta)$ and $f_{2}(\theta) \vee$ $f_{3}(\theta)$. (d) All terms and join result.

maximum value at $v=v_{\max }$, the associated $g_{1}(\theta)=$ $\sup _{v \in[0, \theta]}\left\{f_{1}(v)\right\}$ is as follows:

$g_{1}(\theta)=\sup _{v \in[0, \theta]}\left\{f_{1}(v)\right\}=\left\{\begin{array}{l}f_{1}(\theta) \quad \forall \theta \leq v_{\max } \\ 1 \quad \forall \theta>v_{\max }\end{array}\right.$

Analogously, we can obtain the term $g_{2}(\theta)=$ $\sup _{w \in[0, \theta]}\left\{f_{2}(w)\right\}$ :

$g_{2}(\theta)=\sup _{w \in[0, \theta]}\left\{f_{2}(w)\right\}=\left\{\begin{array}{l}f_{2}(\theta) \quad \forall \theta \leq w_{\max } \\ 1 \quad \forall \theta>w_{\max }\end{array}\right.$

These terms are illustrated in Figure 2(a) and 2(b), respectively. The only term in Equation (4) yet to be analysed is $\left(f_{1}(\theta) \vee f_{2}(\theta)\right)$, which is depicted if Figure 2(c), along with $f_{1}(\theta)$ and $f_{2}(\theta)$. The final join result, which is as in Equation (4), is illustrated in Figure 2(d) in dashed line. It is important to note that the resulting $\mu_{\tilde{F}_{1} \cup \tilde{F}_{2}}(x, \theta)$ is identical to $f_{2}$. Although this result may be surprising, we can get to the same conclusion using the equations by Karnik and Mendel in [3]. We now repeat all operations between the resulting set (depicted in Figure 2(d) in dashed line) and the set illustrated in Figure 1(c). We obtain $g_{2}(\theta), g_{3}(\theta), f_{2}(\theta) \vee f_{3}(\theta)$ and the minimum of all these quantities. Results are illustrated in Figure 3.

It is important to note that the final result displayed in Figure 3(d) is the same that the one presented in [12] (page 493, fig. 


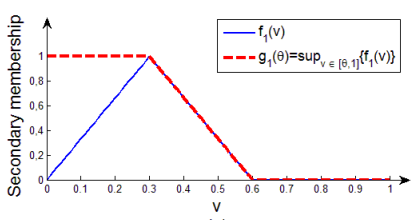

(a)

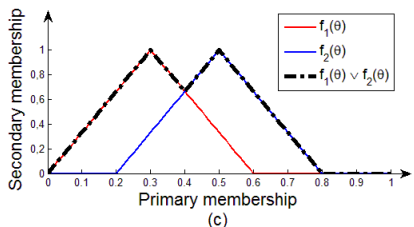

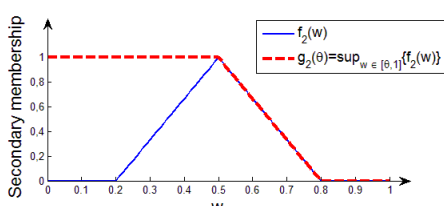

(b)

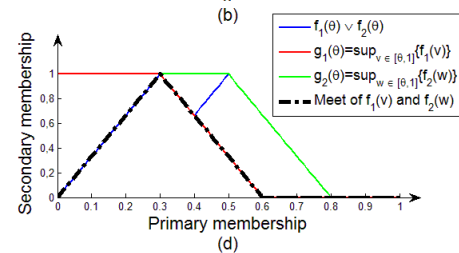

(d)

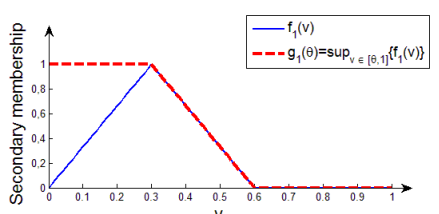

(a)

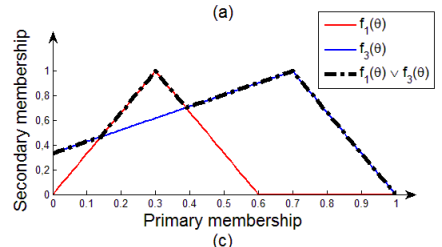

(c)

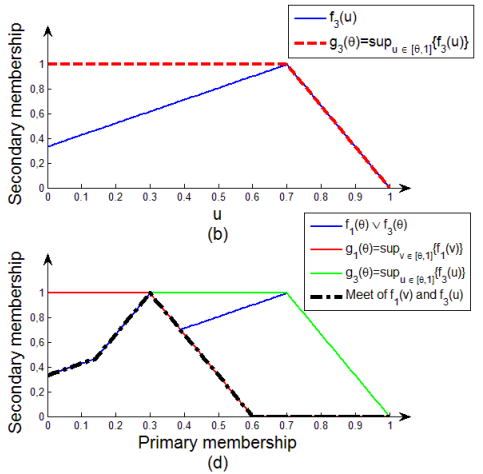

(d)

Fig. 4. (a) $f_{1}(v)$ and $g_{1}(\theta)$. (b) $f_{2}(w)$ and $g_{2}(\theta)$. (c) $f_{1}(\theta), f_{2}(\theta)$ and $f_{1}(\theta) \vee$ $f_{2}(\theta)$. (d) All terms and meet result.

5), and thus, Equation (4) is consistent with the specific case where secondary grades are normal and convex type- 1 sets.

Now we will perform the meet operation on the same three sets depicted in Figure 1. Doing a similar analysis to the one that led to Equations (46) and (47), it can be proven that:

$$
\begin{gathered}
g_{1}(\theta)=\sup _{v \in[\theta, 1]}\left\{f_{1}(v)\right\}=\left\{\begin{array}{l}
1 \quad \theta \leq v_{\text {max }} \\
f_{1}(\theta) \quad \theta>v_{\text {max }}
\end{array}\right. \\
g_{2}(\theta)=\sup _{w \in[\theta, 1]}\left\{f_{2}(w)\right\}=\left\{\begin{array}{l}
1 \quad \theta \leq w_{\text {max }} \\
f_{2}(\theta) \quad \theta>w_{\text {max }}
\end{array}\right.
\end{gathered}
$$

These terms are depicted in Figures 4(a) and 4(b). The only term in Equation (28) yet to be analysed is the second one, $\left(f_{1}(\theta) \vee f_{2}(\theta)\right)$, which is the same as in the join operation and is depicted in Figure 4(c), along with $f_{1}(\theta)$ and $f_{2}(\theta)$. The final meet result, which is as in Equation (28), is illustrated in Figure 4(d).

It is important to note that the resulting $\mu_{\tilde{F}_{1} \cap \tilde{F}_{2}}(x, \theta)$ is identical to $f_{1}$. Although this result may be surprising, we can get to the same conclusion using the equations by Karnik and Mendel in [3]. We now repeat all operations between the resulting set (depicted in Figure $4(\mathrm{~d})$ ) and the set illustrated in Figure 1(c). We obtain $g_{1}(\theta), g_{3}(\theta), f_{1}(\theta) \vee f_{3}(\theta)$ and the minimum of all these quantities. Results are illustrated in Figure 5. It is important to note that the final result displayed in Figure 5(d) is the same that the one presented in [12] (page 495, fig. 7), and thus, Equation (28) is consistent with the specific case where secondary grades are normal and convex type-1 sets.

\section{B. Examples of the Join and Meet operations for GT2FSs with non-convex non-normal secondary grades}

In this section we will apply our approach presented in Equation (4) and Equation (28) to type-2 fuzzy sets whose secondary grades are arbitrary, i.e., secondary grades are neither convex nor normal. The chosen secondary grades to perform this operation are illustrated in Figure 6(a) and 6(b), respectively, in solid lines, along with their terms $g_{1}(\theta)=$ $\sup _{v \in[0, \theta]}\left\{f_{1}(v)\right\}$ and $g_{2}(\theta)=\sup _{w \in[0, \theta]}\left\{f_{2}(w)\right\}$. It is important to note that these terms $g_{1}(\theta)$ and $g_{2}(\theta)$ cannot be

Fig. 5. (a) $f_{2}(w)$ and $g_{2}(\theta)$. (b) $f_{3}(u)$ and $g_{3}(\theta)$. (c) $f_{2}(\theta), f_{3}(\theta)$ and $f_{2}(\theta) \vee$ $f_{3}(\theta)$. (d) All terms and meet result.
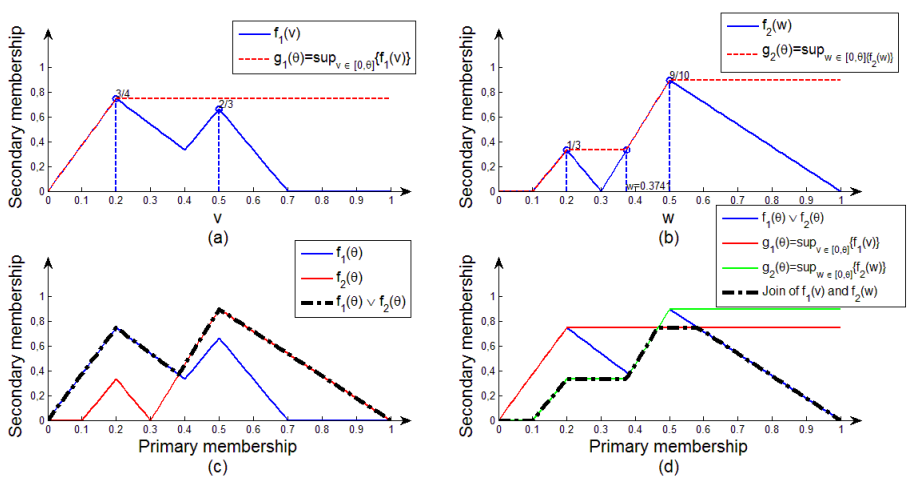

Fig. 6. (a) $f_{1}(v)$ and $g_{1}(\theta)$. (b) $f_{2}(w)$ and $g_{2}(\theta)$. (c) $f_{1}(\theta), f_{2}(\theta)$ and $f_{1}(\theta) \vee$ $f_{2}(\theta)$. (d) All terms and join result.
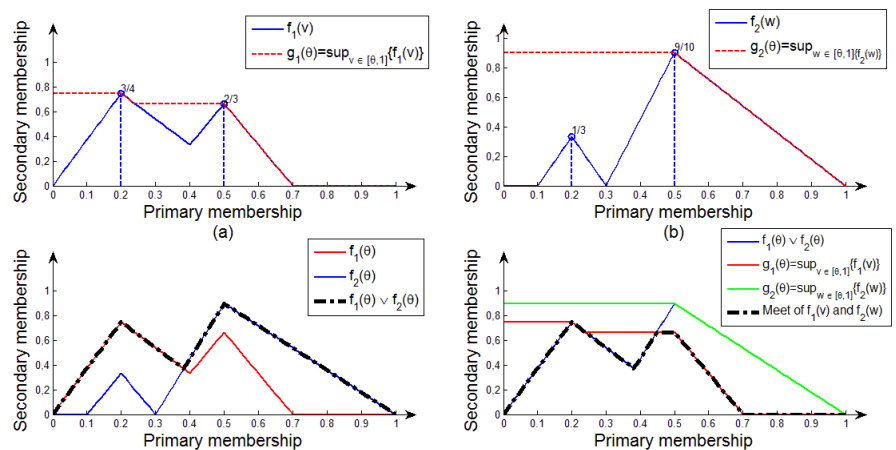

(c)

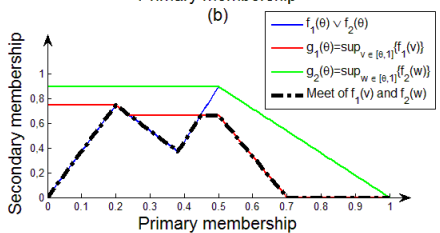

(d)

Fig. 7. (a) $f_{1}(v)$ and $g_{1}(\theta)$. (b) $f_{2}(w)$ and $g_{2}(\theta)$. (c) $f_{1}(\theta), f_{2}(\theta)$ and $f_{1}(\theta) \vee$ $f_{2}(\theta)$. (d) All terms and meet result.

computed using Equations (46) and (47) as the sets are not convex. Figure 6(c) depicts the term $\left(f_{1}(\theta) \vee f_{2}(\theta)\right)$. Figure $6(d)$ illustrates all terms involved along with the final join result.

The procedure to obtain the meet result for the same sets is analogous, but changing the definitions of $g_{1}(\theta)=$ $\sup _{v \in[\theta, 1]}\left\{f_{1}(v)\right\}$ and $g_{2}(\theta)=\sup _{w \in[\theta, 1]}\left\{f_{2}(w)\right\}$. All figures related to the meet operation on the sets depicted in Figure 7(a) and (b) are illustrated in Figure 7. 

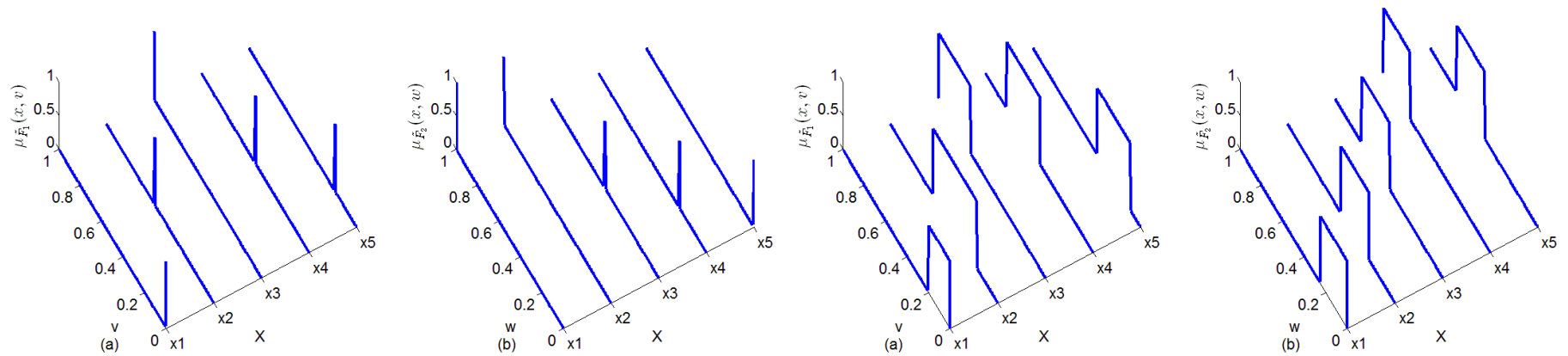

Fig. 8. Sets $\tilde{F}_{1}$ and $\tilde{F}_{2}$ when IT2FSs are equivalent to type-1 sets.

Fig. 10. IVFSs to perform the join and meet operations.
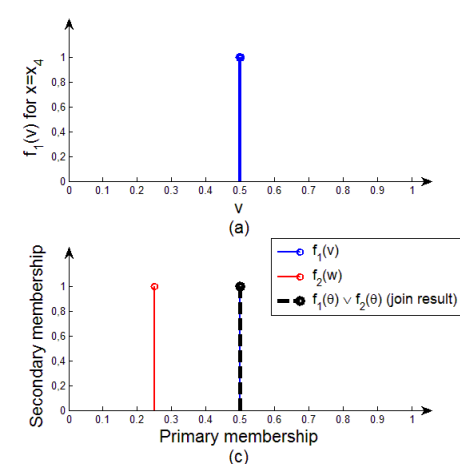

(c)

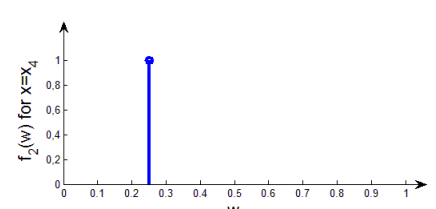

W
(b)

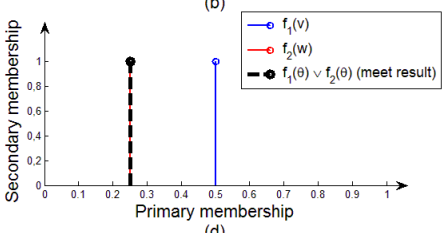

(d)
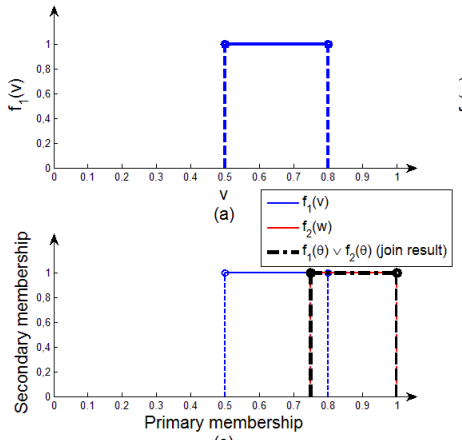

(c)

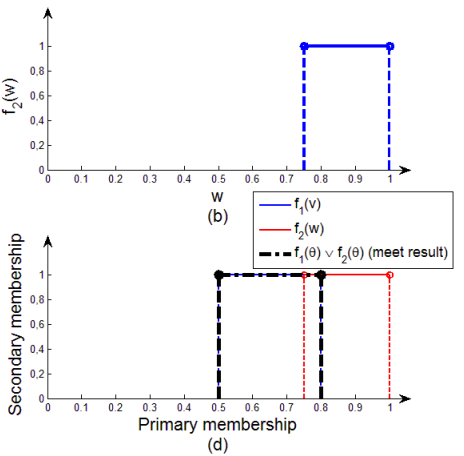

(d)

Fig. 9. (a) $f_{1}(v)$. (b) $f_{2}(w)$. (c)Sets and join result. (d)Sets and meet result.

Fig. 11. (a) $f_{1}(v)$. (b) $f_{2}(w)$. (c)Sets and join result. (d)Sets and meet result.

\section{Examples of Join and Meet Operations for the General Forms of IT2FSs}

In this section, we will focus on the particular case where $f_{1}(v)$ and $f_{2}(w)$ are either 0 or 1 and their supports are closed sets. In other words, we will focus on the general descriptions of IT2FSs, as presented in [1]. We will use Equations (36) and (43) (which are specific versions of Equations (4) and (28)) to compute the join and meet respectively when the secondary grades are either 0 or 1 , to all the cases of general forms of IT2FSs presented in [1]. For simplicity, as in [1], we are going to work with a finite referential set $X$ of cardinal $m$. However, our approach will also be valid for non-finite referential sets. In all cases, we will present the IT2FSs, and we will perform the join and meet operations on the vertical slices placed at $x=x_{4}$.

1) Case A: primary memberships are singletons (type-1 fuzzy sets): The sets to perform the join and meet operations are depicted in Figures 8(a) and (b), respectively. The vertical slices we are going to operate with are depicted in Figure 9(a) and (b); Figure 9(c) illustrates the join operation as given in Equation (36), whereas Figure 9(d) illustrates the meet operation as in Equation (43).

2) Case B: primary memberships are intervals (IVFSs):

The sets to perform the join and meet operations are depicted in Figures 10(a) and (b), respectively. The vertical slices we are going to operate with are depicted in Figure 11(a) and (b); Figure 11(c) illustrates the join operation as given in Equation
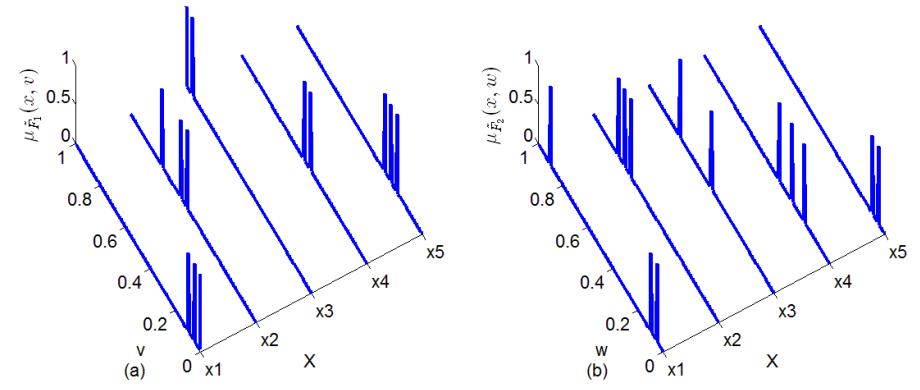

Fig. 12. Multi-singleton IT2FSs to perform the join and meet operations.

(36), whereas Figure 11(d) illustrates the meet operation as in Equation (43).

3) Case C: primary memberships are several singletons: The sets to perform the join and meet operations are depicted in Figures 12(a) and (b), respectively. The vertical slices we are going to operate with are depicted in Figure 13(a) and (b); Figure 13(c) illustrates the join operation as given in Equation (36), whereas Figure 13(d) illustrates the meet operation as in Equation (43).

From [1] it is stated that this example may correspond to a setting in which anonymous users from a website score different objects and/or services within it. In this situation, 


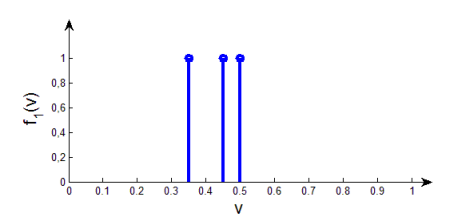

(a)

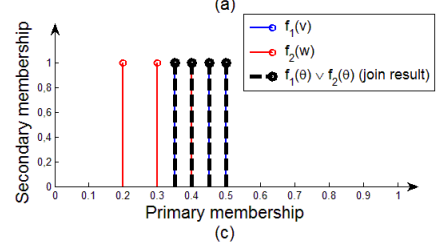

(c)

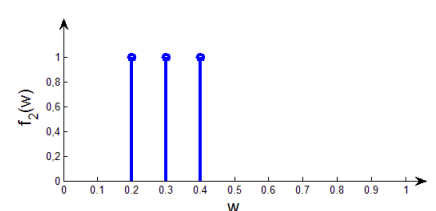

(b)

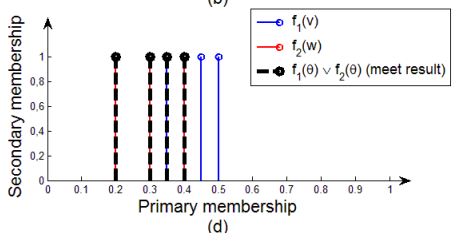

Fig. 13. (a) $f_{1}(v)$. (b) $f_{2}(w)$. (c)Sets and join result. (d)Sets and meet result.
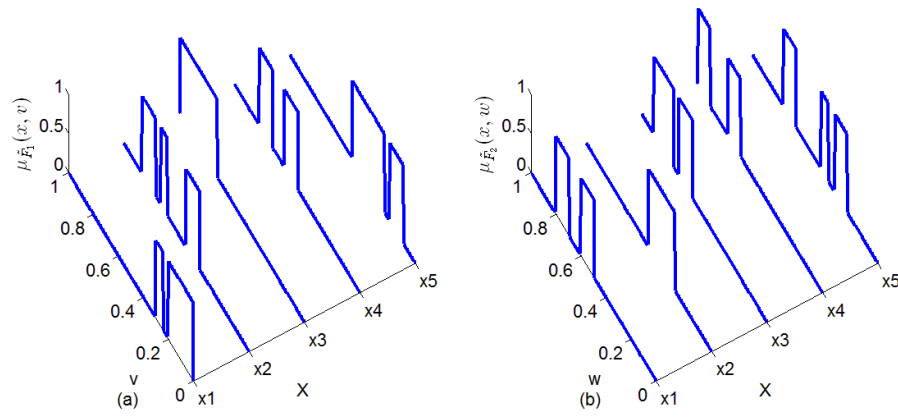

Fig. 14. Multi-IVFSs to perform the join and meet operations.

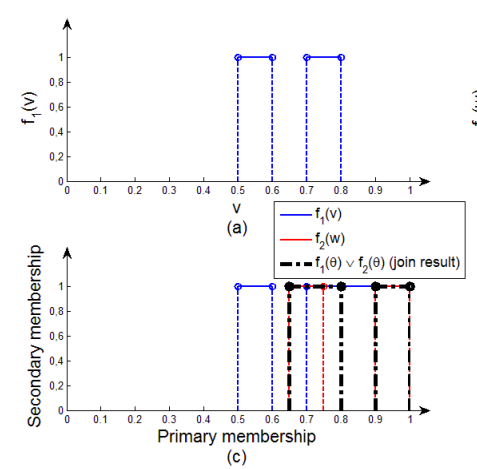

(c)

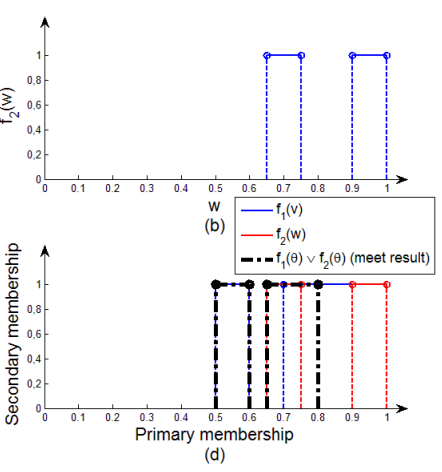

Fig. 15. (a) $f_{1}(v)$. (b) $f_{2}(w)$. (c)Sets and join result. (d)Sets and meet result.

and considering both anonymity and that not every user will score every object, we would obtain sets as in this Case C.

4) Case D: primary memberships are several intervals: The sets to perform the join and meet operations are depicted in Figures 14(a) and (b), respectively. The vertical slices we are going to operate with are depicted in Figure 15(a) and (b); Figure 15(c) illustrates the join operation as given in Equation (36), whereas Figure 15(d) illustrates the meet operation as in Equation (43).

Several intervals could be used as follows: consider we have a FLS which contains the following rules for inputs $x_{1}$ and
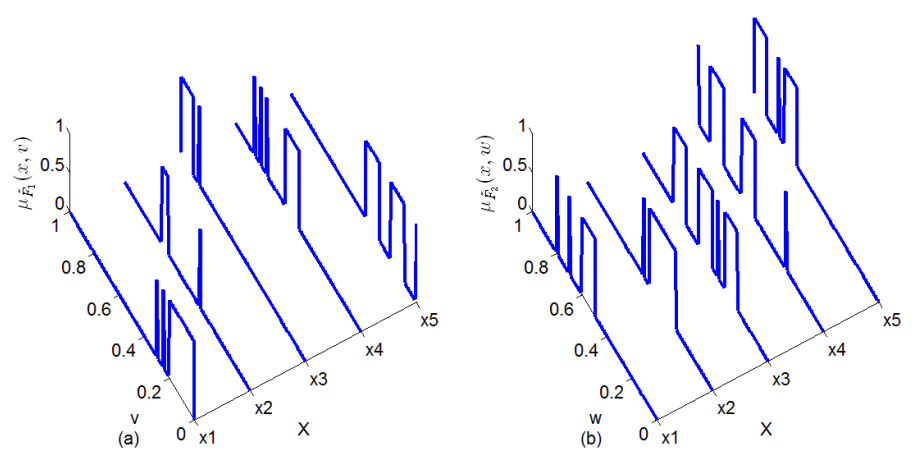

Fig. 16. Sets to perform the join and meet operations.

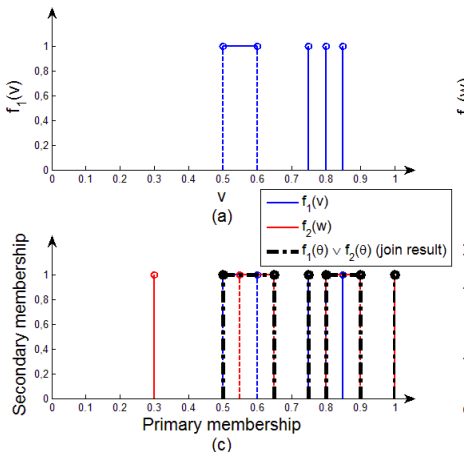

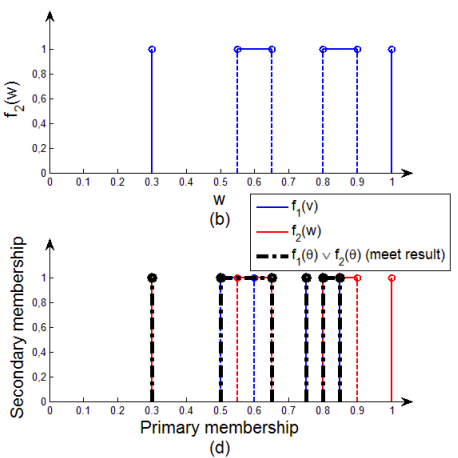

Fig. 17. (a) $f_{1}(v)$. (b) $f_{2}(w)$. (c)Sets and join result. (d)Sets and meet result.

$$
\begin{aligned}
& x_{2}: \\
& \quad R^{l}: \text { IF } x_{1} \text { is HIGH AND } x_{2} \text { is HIGH THEN y is } Y \\
& R^{p}: \text { IF } x_{1} \text { is HIGH AND } x_{2} \text { is LOW THEN y is Y }
\end{aligned}
$$

Where, e.g., $H I G H=[0.9,1]$ and $L O W=[0,0.1]$. This could be modelled using a non-standard rule:

$R^{l^{\prime}}:$ IF $x_{1}$ is HIGH AND $x_{2}$ is HIGH orLOW THEN y is $Y$

Or by using a standard rule with a set EXTREME = $[0,0.1] \cup[0.9,1]$ as follows:

$R^{l^{\prime}}:$ IF $x_{1}$ is HIGH AND $x_{2}$ is EXTREMETHEN y is $Y$

This way the complexity of the FLS can be reduced and remain having a standard rule base.

5) Case E: primary memberships are combinations of singletons and intervals: The sets to perform the join and meet operations are depicted in Figures 16(a) and (b), respectively. The vertical slices we are going to operate with are depicted in Figure 17(a) and (b); Figure 17(c) illustrates the join operation as given in Equation (36), whereas Figure 17(d) illustrates the meet operation as in Equation (43).

Considering again the scoring system in a website, we could use these type of sets when the number of scores provided increases significantly; hence, those regions of the interval 
$[0,1]$ which are very crowded could be replaced by an interval, whereas the most isolated values could remain as singletons.

\section{CONCLUSIONS AND FUTURE WORKS}

In this paper we have presented two theorems to perform the join and meet operations on any GT2FSs having arbitrary secondary grades where the restrictions about the normality or convexity on the secondary grades are no longer required. These results have allowed us to deal with both GT2FSs and the general forms of IT2FSs as presented in [1]. Hence, the paper will help to explore the potential of interval type-2 fuzzy logic systems which use interval type-2 fuzzy sets which are not equivalent to interval valued fuzzy sets.

To complete all the framework related to Fuzzy Logic Systems (FLSs) using these general forms of IT2FSs, future work would focus on the type reduction operation, when the sets involved are IT2FSs which are not equivalent to type-1 sets or IVFSs. We will also explore possible applications that will benefit from using more general forms of IT2FSs.

\section{REFERENCES}

[1] H. Bustince Sola, J. Fernandez, H. Hagras, F. Herrera, M. Pagola, and E. Barrenechea, "Interval type-2 fuzzy sets are generalization of intervalvalued fuzzy sets: towards a wider view on their relationship," 2015.

[2] L. A. Zadeh, "The concept of a linguistic variable and its application to approximate reasoningi," Information sciences, vol. 8, no. 3, pp. 199$249,1975$.

[3] N. N. Karnik and J. M. Mendel, "Operations on type-2 fuzzy sets," Fuzzy sets and systems, vol. 122, no. 2, pp. 327-348, 2001.

[4] S. Coupland and R. John, "Geometric type-1 and type-2 fuzzy logic systems," Fuzzy Systems, IEEE Transactions on, vol. 15, no. 1, pp. 315, 2007.

[5] J. M. Mendel, "On the geometry of join and meet calculations for general type-2 fuzzy sets," in Fuzzy Systems (FUZZ), 2011 IEEE International Conference on. IEEE, 2011, pp. 2407-2413.

[6] H. Tahayori, A. G. Tettamanzi, and G. D. Antoni, "Approximated type2 fuzzy set operations," in Fuzzy Systems, 2006 IEEE International Conference on. IEEE, 2006, pp. 1910-1917.

[7] J. M. Mendel, "General type-2 fuzzy logic systems made simple: a tutorial," Fuzzy Systems, IEEE Transactions on, vol. 22, no. 5, pp. 1162 $1182,2014$.

[8] Q. Liang and J. M. Mendel, "Interval type-2 fuzzy logic systems: theory and design," Fuzzy Systems, IEEE Transactions on, vol. 8, no. 5, pp. $535-550,2000$.

[9] J. M. Mendel, "Uncertain rule-based fuzzy logic system: introduction and new directions," 2001.

[10] C. L. Walker and E. A. Walker, "The algebra of fuzzy truth values," Fuzzy Sets and Systems, vol. 149, no. 2, pp. 309-347, 2005.

[11] P. Hernandez, S. Cubillo, and C. Torres-Blanc, "On t-norms for type-2 fuzzy sets," 2013.

[12] C. Wagner and H. Hagras, "zslicestowards bridging the gap between interval and general type-2 fuzzy logic," in Fuzzy Systems, 2008. FUZZIEEE 2008.(IEEE World Congress on Computational Intelligence). IEEE International Conference on. IEEE, 2008, pp. 489-497. 